\title{
Vascular Pattern of Hyaloid Vessels in Carp Eye
}

\author{
Jun Kohbara, ${ }^{* 1 *^{2}}$ Shiro Murachi, ${ }^{{ }_{1}}$ and Kenji Nanba ${ }^{* 1}$ \\ (Accepted June 17, 1986)
}

\begin{abstract}
The vascular architecture of hyaloid vessel in carp eye was investigated using the trypsin digestion method. At the center of the optic disk, the hyaloid arteries branch off in radial directions and proceed to the peripheral region of the retina. The branching of hyaloid arteries was divided roughly into two types. One is the dichotomous ( $Y$-shaped) branching having two branches of equal diameter. The other is the side-arm branching which comes off at right angles from thick arteries. It was suggested that the former plays an important role in the blood transportation to the peripheral region. On the other hand, the latter contributes to extending the region of blood supply by making capillary plexus between the thick arteries. The capillaries come together again and form veins between the thick arteries. Both the capillaries and the veins anastomose to the annular vessel which run circularly on the ora terminalis. In addition, in contrast with almost all vessels anastomosing to the annular vessel, some capillaries seen in the neighborhood of the optic disk come back again to this disk. Thus, there are two efferent blood streams in the hyaloid vessels.
\end{abstract}

Studies on the retinal vascular architecture have been performed using various methods. Among them, the most widespread technique to visualize the vessels is the intravascular injection from the aorta or the heart using ink or latex..$^{1-5)}$ However, this useful method also has some limitations. The solicitous points of this technique are occurrence of vessel occlusion by large particles of injected substances or the vessel rupture due to the too high pressure during the injection. In both cases, there is the possibility that the vessel situating at the distal part from the troubled portion is not observed. Moreover, no information is obtainable about the vessel wall by injection technique. To avoid these troubles, the trypsin digestion method by Kuwabara and $\operatorname{Cogan}^{6)}$ is considered to be very useful. So, we tried to clarify the vascular pattern of hyaloid vessels of carp eye using this digestion method.

\section{Materials and Methods}

Six carp (Cyprinus carpio, $22.5-27.0 \mathrm{~cm}$ in total length and $142-234 \mathrm{~g}$ in body weight) were used in the present study. The enucleated eyes without the cornea and the lens were fixed in $20 \%$ formalin solution for several days. The trypsin digestion was performed by the procedure of Kuwabara and Cogan. ${ }^{6)}$ This procedure is as follows: the fixed eyes were washed overnight in running water and incubated in $0.1 \mathrm{M}$ Tris buffer solution containing $3 \%$ trypsin for about $3 \mathrm{~h}$ at $37^{\circ} \mathrm{C}$. After incubation, the eyes were transferred into clean water. The hyaloid vessels and the inner limiting membrane were able to peel off together under careful manipulation, because the vessels attach intimately to the inner limiting membrane. The specimen peeled off from the globular eye had a bowl shape, therefore, in water four or five small cuts were made at the peripheral region spreading on a flat slide glass. After drying the specimens were stained with PAS and hematoxylin.

\section{Results and Discussion}

The vascular structure of hyaloid vessels were well preserved and they correspond to the image obtained from the ophthalmoscopic observation. ${ }^{7)}$ As the hyaloid vessels of carp exist on and attach themselves to the inner limiting membrane, the patterns of hyaloid vessels seem to be protected from the mechanical damage by supporting of inner limiting membrane.

The optic artery penetrating the optic nerve and appearing on the fundus of eye branches off in all directions at the center of the optic disk (Fig. 1). On the eye fundus, two branching styles are observed in the hyaloid vessels. One is the dicho-

*. Faculty of Applied Biological Science, Hiroshima University, Fukuyama 720, Japan (神原淿, 村地四郎,

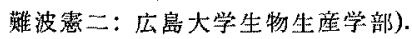

*2 Present Address: Faculty of Fisheries, Mie University, Tsu 514, Japan (三重大学水挫学部). 


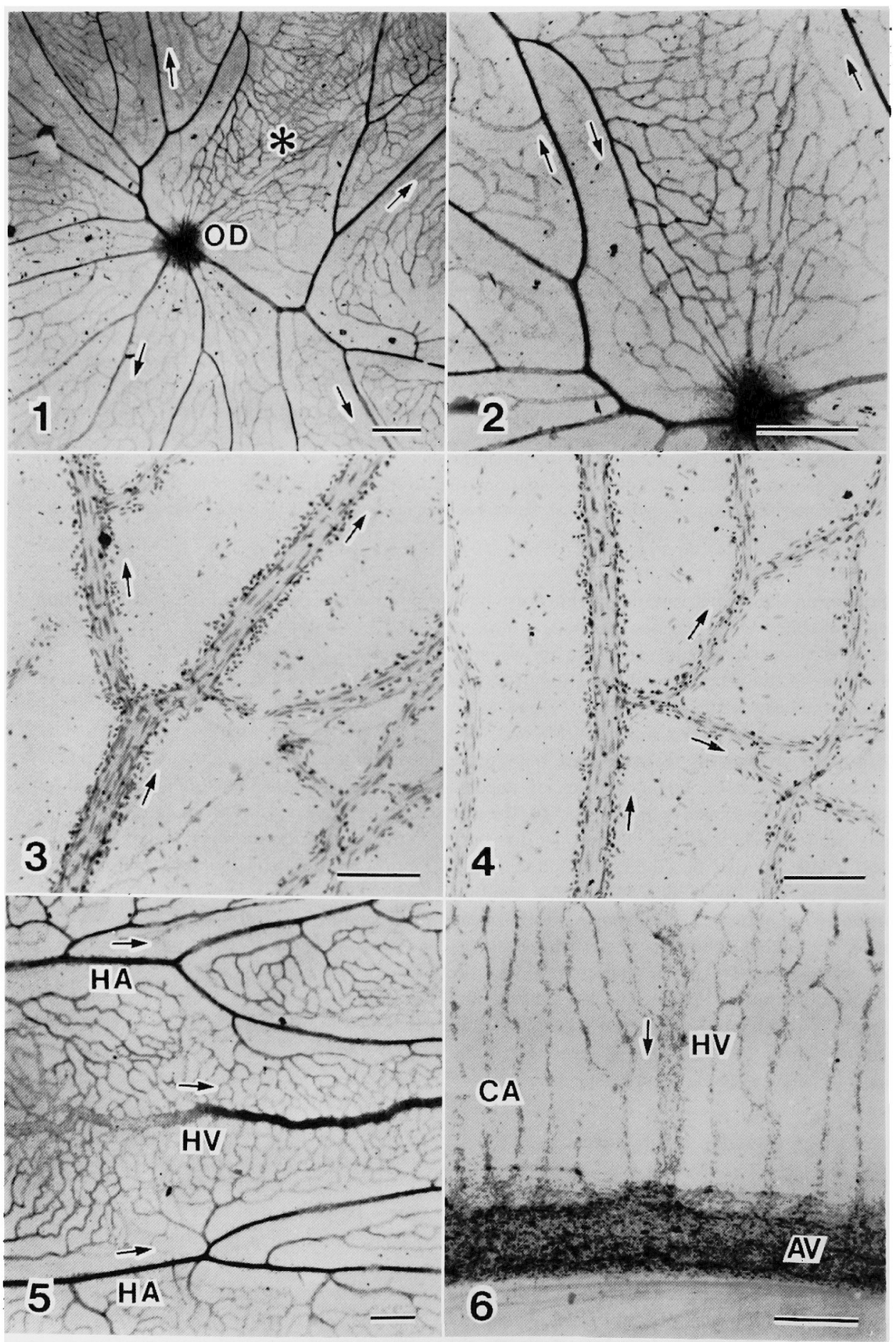


tomous branching of thick arteries (Fig. 3). These two branches are equal in diameter. The thick arteries running on the retina repeat divergence and become slenderer toward periphery. Finally, they form capillary plexus at the peripheral regions of the retina. The other is the side-arm branching which comes off at right angles from thick arteries and also form capillary plexus between the thick arteries (Fig. 4). Between the thick arteries, these capillaries come together again and form veins at the peripheral region of the retina (Fig. 5), Both capillaries and veins anastomose to the annular vessel running circularly on the ora terminalis (Fig. 6). Furthermore, almost all vessels anastomose to the annular vessel, but some capillaries distributing in the neighborhood of the optic disk come back again to the optic disk (Fig. 2). Thus, there are two efferent blood streams in the hyaloid vessels.

The vascular pattern of carp hyaloid vessels is quite similar in branching styles to that of the retinal vessels of human beings. Also in human being, the dichotomous branchings and the right angle ones are observed in the retinal vessels, ${ }^{6)}$ and these two styles are said to have different functional significance. The dichotomous branching which having two arms of equal diameter often occur in the thick arteries. These thick arteries play an important role in the blood transportation to the peripheral region of the retina. On the other hand, the right angle branchings making capillaries between the thick arteries contribute to extending the region of blood supply. According to Kuwabara and Cogan, ${ }^{\text {2 }}$ the net of human retinal vessels might be effective in equalization of capillary blood pressure throughout the entire retina. Also, this explanation may be applicable to the case of the hyaloid vessels of carp eye.

The abundant capillaries of hyaloid vessels may be favorable to the metabolism of retinas, in addition to the vascular supply by the choroidal vessels in the opposite side of the retina. This dual vascular supply seems to be more effective than the ocular vascular system of fishes having falciform process in their eyes. On the other hand, the erythrocytes flowing in the hyaloid vessels seems to be an obstacle to the light reception and analysis from the standpoint of visual acuity. For example, the region of fovea, the most important and acutest portion in the human or mammalian retina, is lacking in the vascularity by retinal vessels and maintained only by choroidal vessels. On the contrary, Copeland ${ }^{3)}$ reported that the area centralis of Fundulus is maintained by abundant capillaries of hyaloid vessel. These peculiar capillaries are not observed in the carp hyaloid vessels of the present study. Thus, some complicated problems still remain. However, it is very interesting that the hyaloid vessels are often present in the eye of lazy fishes such as cyprinoid, gobioid or labroid fish living in tubid water or at the bottom of water. ${ }^{13}$

As for the veins of hyaloid vessels, necessity for their existence is uncertain. If the annular vessel is considered as the final and collecting vein in hyaloid vessels, the hyaloid veins seems to be not always necessary. As a matter of fact, both the capillaries and the veins anastomose with the annular vessel. Is it essential that blood vessels dividing finally into capillaries have a character of gathering together and form veins? The existence of veins may be inevitable in the closed blood-vascular system. To clarify this problem, further study on the fine structure and the permeability of hyaloid vessel wall is necessary.

\section{Acknowledgments}

We wish to thank Prof. I. Hanyu, Faculty of Agriculture, University of Tokyo, and Prof. M.

\section{Explanation of Figures}

All arrows in these figures indicate the direction of blood flow.

Fig. 1. General aspect of hyaloid vessels of carp eye demonstrated by whole flat-mounted method after trypsin digestion. OD: optic disk. Asterisk is explained in Fig. 2. Bar $=0.5 \mathrm{~mm}$.

Fig. 2. Magnified figure of the region indicated by the asterisk in Fig. 1. Some capillaries distributing in the neighborhood of the optic disk return to the optic disk. Bar $=0.5$ $\mathrm{mm}$.

Fig. 3. Dichotomous branching of thick hyaloid artery with two arms of equal diameter. Bar $=0.1 \mathrm{~mm}$.

Fig. 4. Side arm branching from thick hyaloid artery. Bar $=0.1 \mathrm{~mm}$.

Fig. 5. Hyaloid vein (HV) between the two thick hyaloid arteries (HA). Bar $=0.1 \mathrm{~mm}$.

Fig. 6. Both veins (HV) and capillaries (CA) anastomose to the annular vessel (AV). Bar $=0.1 \mathrm{~mm}$. 
Oguri, Faculty of Agriculture, Nagoya University, for their critical readings of this manuscript.

\section{References}

1) I. Hanyu: Nippon Suisan Gakkaishi, 25, 595-613 (1959).

2) I. Hanyu: Nippon Suisan Gakkaishi, 25, 614-619 (1959).

3) D. E. Copeland: Exp. Eye Res., 19, 583-589
(1974).

4) D. E. Copeland and A. T. Fitzjarrell: Exp. Eye Res., 21, 515-521 (1975).

5) D. E. Copeland: Exp. Eye Res., 22, 169-179 (1976).

6) T. Kuwabara and D. G. Cogan: Arch. Ophthal., 64, 904-911 (1960).

7) S. Murachi, J. Kohbara, H. Yamada, S. Sakurai, and K. Nanba: Anim. Eye Res., 5, 41-48 (1986). 